\title{
Produção e espessura da serapilheira na borda e interior de fragmentos florestais de Mata Atlântica de diferentes tamanhos ${ }^{1}$
}

\author{
RITA DE CÁSSIA QUITETE PORTELA² e FLAVIO ANTONIO MAËS DOS SANTOS ${ }^{3,4}$
}

(recebido: 29 de dezembro de 2005; aceito: 26 de abril de 2007)

\begin{abstract}
Litter production and accumulation in the edge and interior of Atlantic Rain forest fragments of different sizes). Nowadays, forests fragmentation is one of the main impacts in the natural communities and can lead to extinction of many species and modifications in many ecological processes. The present work aimed to evaluate the influence of the size and the edge of forest fragments in the production and accumulation of litter and in the canopy openness of four different-size forest fragments of the Atlantic Forest. The litter production was bigger in the larger fragment $\left(8.90 \mathrm{tha}^{-1} \mathrm{year}^{-1}\right.$ in the interior and 8.76 $\mathrm{t} \mathrm{ha}^{-1}$ year ${ }^{-1}$ in the edge) than in the three smaller fragments $\left(7.29 \mathrm{t} \mathrm{ha}^{-1}\right.$ year ${ }^{-1}$ in the interior and $7.27 \mathrm{t} \mathrm{ha}^{-1} \mathrm{year}^{-1}$ in the edge), however the thickness of the litter layer deposited on the ground and the canopy openness of the forest had no relation with the size of the fragments. No difference was found in the production and accumulation of litter and canopy openness between areas of edge and interior. The dry season had a direct influence in the increase of the litter production. Concerning the fragment area, we can infer that the difference between litterfall and accumulation on the ground might be related with the greater decomposition rates in the larger fragment, due to the higher humidity. No relation between litter production and canopy openness was found, probably because of the great spatial heterogeity caused by the frequency of gap formation and structural complexity and deciduousness of the forest.
\end{abstract}

Key words - Atlantic rain forest, canopy openness, edge effects, forest fragments, litter

RESUMO - (Produção e espessura da serapilheira na borda e interior de fragmentos florestais de Mata Atlântica de diferentes tamanhos). A fragmentação florestal é um dos principais impactos antrópicos a comunidades naturais da atualidade, podendo levar à extinção muitas espécies e também alterar muitos processos ecológicos. O presente trabalho teve como objetivo avaliar a influência do tamanho e da borda de fragmentos florestais na produção e espessura da serapilheira e na abertura do dossel de quatro fragmentos florestais de Mata Atlântica de diferentes tamanhos. A produção de serapilheira foi maior no maior fragmento $\left(8,90 \mathrm{t} \mathrm{ha}^{-1}\right.$ ano $^{-1}$ no interior e na borda $8,76 \mathrm{t} \mathrm{ha}^{-1}$ ano $\left.^{-1}\right)$, do que nos três fragmentos pequenos $\left(7,26 \mathrm{t} \mathrm{ha}^{-1}\right.$ ano $^{-1}$ na $^{-}$ interior e 7,27 t ha- $\mathrm{ano}^{-1}$ na borda), já a espessura da camada de serapilheira depositada sobre o solo e a abertura do dossel da floresta não apresentaram relação com o tamanho do fragmento. Não foi encontrada diferença entre a produção e espessura de serapilheira e a abertura do dossel entre áreas de borda e de interior. A estação seca teve influência direta no aumento da produção de serapilheira. Com relação à área do fragmento, podemos inferir que a diferença entre queda e espessura de serapilheira deve estar relacionada com diferenças de taxa de decomposição nesses fragmentos, sendo maior no fragmento grande devido à maior umidade. Não houve relação entre a produção de serapilheira e a abertura do dossel, provavelmente devido à grande heterogeneidade espacial causada pela freqüência de clareiras e complexidade estrutural e deciduidade da floresta.

Palavras-chave - abertura do dossel, efeito de borda, fragmentos florestais, mata atlântica, serapilheira

\section{Introdução}

A queda e o acúmulo de serapilheira é um fator que afeta a estrutura e a dinâmica da comunidade de plantas de muitas maneiras diferentes (Faccelli \& Pickett 1991). A serapilheira pode influenciar mudanças

\footnotetext{
1. Parte da dissertação de mestrado do primeiro autor

2. Programa de Pós-Graduação em Biologia Vegetal, Departamento de Botânica, Instituto de Biologia, Universidade Estadual de Campinas

3. Universidade Estadual de Campinas, Instituto de Biologia, Departamento de Botânica, Caixa Postal 6109, 13083-970 Campinas, SP, Brasil

4._Autor para correspondência: fsantos@unicamp.br
}

no ambiente físico, como alterações na disponibilidade de nutrientes, na temperatura do solo e na disponibilidade de luz (Faccelli \& Pickett 1991), além de ser um importante fator no entendimento da ciclagem de nutrientes em ecossistemas florestais (Rogers 2002, Vasconcelos \& Luizão 2004). A serapilheira é a maior fonte de aporte de nutrientes no solo de uma floresta (Vitousek 1984). As influências na comunidade vegetal por parte da queda e acúmulo de serapilheira também são inúmeras, relacionadas com a germinação de sementes (Faccelli \& Pickett 1991), com o controle da emergência (Reader 1993) e com o estabelecimento de plântulas (Faccelli \& Pickett 1991, Drake \& Pratt 2001, García-Guzmán \& Benítez-Malvido 2003, Reader 1993). 
Em um estudo feito em floresta semidecídua no interior do Estado de São Paulo, foram encontradas mais plântulas emergindo em áreas onde a serapilheira foi retirada do que em áreas onde a serapilheira foi mantida (Santos \& Válio 2002). Outro exemplo, também experimental, realizado na Amazônia Central, indicou que áreas com maior acúmulo de serapilheira influenciaram no aumento de herbivoria e infestação por fungos em plântulas (García-Guzmán \& BenítezMalvido 2003). Esses exemplos confirmam a importância da serapilheira na organização de comunidades vegetais (Faccelli \& Faccelli 1993). Respostas de diferentes populações a essas mudanças no ambiente, junto com o efeito dessas mudanças na performance de outras populações, podem ter importante influência na estruturação de uma comunidade biológica (Faccelli \& Pickett 1991).

Florestas tropicais possuem estrutura e composição florística muito complexas, o que afeta diretamente a produção de serapilheira, decomposição e liberação de nutrientes para o meio (Alvarez-Sánchez \& Guevara 1999, Vasconcelos \& Luizão 2004). A produção de serapilheira de uma área depende primeiramente da produtividade da comunidade de plantas, e o principal fator abiótico determinante dessa produção é o clima, sendo a precipitação e a temperatura os principais componentes (Faccelli \& Pickett 1991).

A variação temporal na queda de serapilheira é correlacionada com a quantidade de chuvas e tem sido relatada para quase todas as florestas tropicais do mundo. Alguns estudos em florestas sazonais observaram queda contínua, de intensidade variável, com um máximo de queda no final da estação seca (Edwards 1977, KunkelWestphal \& Kunkel 1979, Morellato 1992, Haines \& Foster 1997), podendo aumentar cerca de 2 a 2,5 vezes na estação seca quando comparada à úmida (Sizer et al. 2000). O máximo de queda de folhas na estação seca está aparentemente relacionado à redução de perda de água pelas árvores em florestas estacionais (Haines $\&$ Foster 1997). Entretanto, outros estudos em florestas úmidas sempre verdes encontraram a maior queda de serapilheira no período mais chuvoso, certamente influenciada por períodos de fortes chuvas (Edwards 1977, Moraes et al. 1999, Nascimento 2005). Esses períodos de chuvas fortes provocam maior queda de material lenhoso, levando a um aumento de formação de clareiras e, conseqüentemente, um aumento da abertura do dossel (Martínez-Ramos et al. 1988).

Com relação à variação espacial, o acúmulo de serapilheira é variável, com áreas completamente sem acúmulo (áreas de erosão e zona das raízes de uma clareira), a áreas com grande acúmulo de serapilheira (zona da copa de uma clareira, bases de áreas inclinadas e sobre palmeiras acaules) (Alvarez-Sánchez \& Guevara 1999, Santos \& Válio 2002, García-Guzmán \& Benítez-Malvido 2003).

Com a fragmentação de habitats, várias mudanças ocorrem nos fatores abióticos e bióticos dos remanescentes. Com relação aos abióticos, as principais alterações são no microclima, como por exemplo, aumento da temperatura do ar, aumento da intensidade dos ventos e diminuição da umidade do ar e do solo (Kapos et al. 1997, Siqueira et al. 2004). Outra mudança importante que acaba por acentuar as mudanças no microclima é o aumento da intensidade luminosa no interior dos fragmentos devido ao aumento da abertura do dossel (Kapos 1989). Essas alterações microclimáticas geram diversas mudanças na comunidade vegetal dos remanescentes, tais como o aumento das taxas de mortalidade de árvores na proximidade da borda (Laurance et al. 2001), baixa taxa de germinação em fragmentos florestais (Bruna 2002), diminuição da riqueza e abundância de espécies lenhosas e herbáceas na proximidade da borda (Fontoura et al. 2006), diminuição da densidade de plântulas em fragmentos (Benítez-Malvido \& Martinez-Ramos 2003) e alterações na produção de serapilheira (Scariot 2000, Sizer et al. 2000, Vasconcelos \& Luizão 2004). De acordo com Scariot (2000) e Sizer et al. (2000), após 10 a 15 anos de isolamento, fragmentos isolados na Amazônia Central tendem a apresentar queda de serapilheira semelhante àquela observada em áreas de floresta contínua, provavelmente devido à redução dos efeitos de borda, à regeneração da matriz e ao crescimento de lianas e arbustos na borda. Com relação à produção diferenciada de serapilheira entre a borda $\mathrm{e}$ o interior de fragmentos, Sizer et al. (2000) encontraram taxa de queda de serapilheira maior nos primeiros $10 \mathrm{~m}$ de borda, imediatamente após a formação desta, tornando-se iguais após 1,5 anos do isolamento de um fragmento de 100 ha na Amazônia Central. Vasconcelos \& Luizão (2004) estudando a mesma região, encontraram aumento da produção de serapilheira na borda após 10-20 anos de isolamento. Esse aumento, tanto da produção como do acúmulo de serapilheira nas bordas de fragmentos, parece estar relacionado com o estresse hídrico que algumas espécies vegetais sofrem nesses ambientes, com conseqüente liberação de folhas (Kapos et al. 1993). Outra razão possível é a maior lentidão na decomposição nesse tipo de ambiente (Kapos et al. 1993). Já em um estudo realizado em um fragmento bem conservado de Mata Atlântica, com 
aproximadamente 40 anos de isolamento, no Estado do Rio de Janeiro, foi encontrada uma relação inversa, ou seja, menor produção na borda que no interior (Nascimento 2005). Nesse estudo, a fração folhas, que apresenta a maior contribuição percentual da serapilheira produzida, foi afetada negativamente pela borda, o que pode ser reflexo da redução na densidade de árvores de grande porte do interior para a borda desse fragmento (Nascimento 2005). Essa diferença encontrada entre os estudos na Amazônia e na Mata Atlântica pode ser devida à diferença de idade das bordas e histórico da fragmentação.

Este trabalho teve como objetivo responder às seguintes questões: (1) a produção e a espessura da camada de serapilheira diferem entre fragmentos florestais de Mata Atlântica de diferentes tamanhos? (2) diferem entre a borda e o interior de fragmentos? (3) existe relação da produção de serapilheira com as estações do ano? e (4) existe relação da abertura do dossel com a produção de serapilheira?

\section{Material e métodos}

Área de estudo - Foram estudados um fragmento grande e três fragmentos pequenos. $\mathrm{O}$ fragmento grande estudado foi a Reserva Estadual do Morro Grande ( 9.400 ha) localizado no Município de Cotia, nos bordos do Planalto Atlântico de São

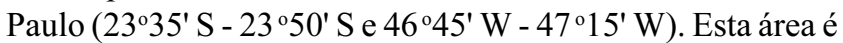
coberta por floresta secundária e madura e protegida há cerca de 80 anos. As áreas de estudo na Reserva possuem altitude variando de 898 a $979 \mathrm{~m}$. Os três fragmentos de floresta secundária estão localizados no município de Ibiúna, possuem cerca de 50 anos, tamanho pequeno (14, 18 e $30 \mathrm{ha}$ ), isolados, de forma irregular e a altitude variando de 904 a $1.003 \mathrm{~m}$. Distam entre si 4,3 km e cerca de 13,7 km da Reserva.

A paisagem onde se situam os fragmentos está localizada a sudeste da Reserva e é dominada por áreas abertas, que cobrem 58\% da paisagem (campos de agricultura - 33\%, áreas com construções rurais ou áreas urbanas - $15 \%$, e vegetação nativa em estádio inicial de sucessão - 10\%) (Pardini et al. 2005). Florestas nativas (e.g., vegetação secundária) cobrem $31 \%$ da paisagem, e plantação de pinus e eucalipto, $7 \%$ (Pardini et al. 2005). Tanto a reserva como a paisagem fragmentada possuem condições geológicas, geomorfológicas, climáticas e vegetacionais similares, o que as torna favoráveis para comparação (Uezu et al. 2005).

Os fragmentos pequenos encontram-se, em sua maior parte, em estádio médio de sucessão, que foi definido como vegetação arbórea, padrão predominantemente homogêneo e com emergentes ocasionais (Metzger et al. 2002). Esse estádio foi determinado através de interpretação de fotografias aéreas do ano de 2000 na escala de 1:10.000. Os três fragmentos possuem mais de $40 \%$ de sua área distando mais de $40 \mathrm{~m}$ da borda (Metzger et al. 2002). Geralmente estas áreas possuem maior estabilidade com relação ao restante do fragmento em termos de variação de temperatura, umidade e vento (Siqueira et al. 2004).

O clima é temperado quente e úmido, do tipo $C f a$ de Köppen (Köppen 1948). A temperatura anual varia de $11^{\circ} \mathrm{C}$ a $27^{\circ} \mathrm{C}$ e a precipitação média anual fica em torno de $1.400 \mathrm{~mm}$, com os meses mais frios e secos entre abril e agosto, mas sem déficit hídrico (Sabesp 1997, figura 1). Predominam na região os Argissolos Vermelho-Amarelos, Latossolos VermelhoAmarelos e Cambissolos (Ross \& Moroz 1997). Nos anos de coleta $(2000$ e 2001$)$ a temperatura média anual foi de $18,9^{\circ} \mathrm{C}$ e $19,5^{\circ} \mathrm{C}$, respectivamente, e a diferença entre os meses mais frio e mais quente foi de $8{ }^{\circ} \mathrm{C}$ em ambos os anos (figura 1). A média anual de precipitação para os anos de coleta ficou em $1.244 \mathrm{~mm}$, sendo os meses com precipitação menor do que $100 \mathrm{~mm}$ : março a agosto e outubro de 2000 e março a setembro de 2001. A vegetação da região é uma transição entre Mata Atlântica Costeira e a Mata Atlântica Semi-decídua, sendo classificada como "Mata Atlântica Baixo Montana" (Oliveira Filho \& Fontes 2000). Pesquisas florísticas na região encontraram alta riqueza de espécies arbóreas (362 espécies com DAP $>5 \mathrm{~cm}$ ), com dominância das famílias Myrtaceae (79 espécies), Lauraceae (38) e Fabaceae (31) (Bernacci et al. 2004).

No fragmento grande (Reserva - 9.400 ha) foram feitas três replicações para cada local (borda e interior), devido à ausência, nos Municípios de Cotia e Ibiúna, de outras áreas florestais extensas bem preservadas. As áreas do fragmento grande serão denominadas, aqui, áreas 1, 2 e 3. Em cada

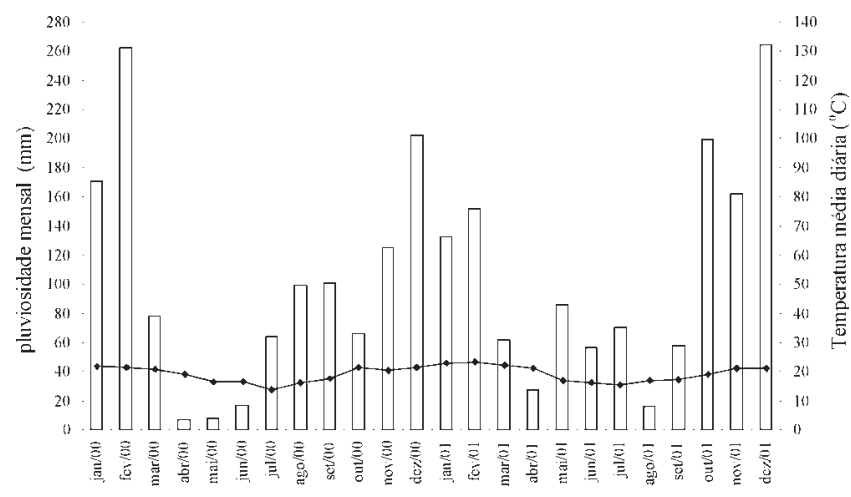

Figura 1. Temperatura média diária e pluviosidade mensal dos anos de 2000 e 2001 do Município de Ibiúna, SP. Dados do IAC de Campinas (CIIAGRO). Posto de coleta fica a $26 \mathrm{~km}$ em linha reta da Reserva Estadual do Morro Grande. ( $\square=$ pluviosidade mensal; $\rightarrow-=$ temperatura média diária).

Figure 1 . Daily average temperature and monthly rainfall in 2000 and 2001 in the city of Ibiúna, SP. Data from IAC of Campinas (CIIAGRO). Place of collection is $26 \mathrm{~km}$ straightline of the "Reserva Estadual do Morro Grande". ( $\square=$ average monthly rainfall; $\rightarrow-=$ average daily temperature). 
fragmento pequeno foi instalada uma parcela na borda e outra no interior. No total foram instaladas seis parcelas no fragmento grande (três na borda e três no interior) e duas em cada fragmento pequeno (uma na borda e uma no interior), totalizando 12 parcelas.

Desenho Experimental - Nos fragmentos pequenos, as parcelas $(10 \times 100 \mathrm{~m})$ foram posicionadas a $5 \mathrm{~m}$ da borda, definida como o ponto até onde o dossel da floresta se estende (Fox et al. 1997), e outra, de mesma área, no interior, a $100 \mathrm{~m}$ da parcela da borda. No fragmento grande, as parcelas $(10 \mathrm{x}$ $100 \mathrm{~m}$ ) também foram posicionadas a $5 \mathrm{~m}$ da borda, mas a distância média entre a parcela da borda e sua correspondente de interior foi 4,1 km. A distância média entre as parcelas da borda foi $2,5 \mathrm{~km}$ e entre as parcelas do interior, foi de $1,8 \mathrm{~km}$. Essas parcelas tiveram o seu comprimento de $10 \mathrm{~m}$ perpendicular à borda para reduzir possíveis interferências do gradiente borda - interior, que, segundo a literatura, podem se estender por $13 \mathrm{~m}$ até $5 \mathrm{~km}$ de borda (Kapos 1989, WilliamsLinera 1990, Laurance \& Yensen 1991, Kapos et al. 1993, Matlack 1993, Kapos et al. 1997, Didham \& Lawton 1999, Gehlhausen et al. 2000).

Coleta de serapilheira - A serapilheira produzida foi coletada a cada três meses, durante um ano, sempre na primeira semana do mês, iniciando em setembro de 2000 (coletas feitas em dezembro de 2000, março de 2001, junho de 2001 e setembro de 2001), em 10 pontos sorteados em cada parcela. Para a escolha desses pontos, a parcela foi quadriculada de $1 \mathrm{em} 1$ $\mathrm{m}$, formando um par de eixos cartesianos, e os 10 pontos foram sorteados para cada uma. A serapilheira foi coletada por meio de coletores de $0,5 \times 0,5 \mathrm{~m}$, distantes $0,2 \mathrm{~m}$ do solo para evitar a decomposição da matéria acumulada ao longo dos três meses. Todas as frações da serapilheira foram consideradas, sem descarte de qualquer material encontrado dentro dos coletores. Os coletores foram construídos em madeira e tela de tecido organza, que permite a drenagem da água, e permaneceram na mesma posição durante toda a coleta de dados. Quatro coletores foram virados por animais e tiveram a serapilheira que ainda havia no interior descartada, e recolocados na posição original para coleta de dados no período seguinte. Cada amostra de serapilheira foi acondicionada em saco de papel, depois colocada em estufa a $80{ }^{\circ} \mathrm{C}$ por 48 horas e pesada.

Os dados de produtividade (PS) tiveram que ser transformados para raiz quadrada para normalizarem (D'Agostino - Pearson, $g l=2, P=0,191$ ), pois apresentaram distribuição de Poisson (Zar 1999), quando não transformados.

Espessura da serapilheira - A espessura da camada de serapilheira foi medida por meio de uma régua de $30 \mathrm{~cm}$ em 50 pontos aleatórios dentro de cada parcela $(10 \times 100 \mathrm{~m})$. Para escolha dos pontos, a parcela foi quadriculada de $1 \mathrm{em} 1 \mathrm{~m}$, formando um par de eixos cartesianos, e os 50 pontos foram sorteados. Os dados foram coletados em junho e setembro de 2001. Cada um dos 50 pontos foi considerado uma réplica dentro da parcela.
Os dados de espessura tiveram que ser transformados para logaritmo na base 10 para normalizarem (D'Agostino Pearson, $g l=2, P=0,153)$, pois apresentaram um desvio na simetria (Zar 1999), quando não transformados.

Abertura do dossel - Foram coletadas quatro medidas da abertura do dossel em 5 pontos sorteados dentro de cada parcela em setembro e dezembro de 2001. Para a escolha dos pontos, a parcela foi quadriculada de $1 \mathrm{em} 1 \mathrm{~m}$, formando um par de eixos cartesianos, e os 5 pontos foram sorteados. Foi utilizado um densiômetro esférico côncavo e as medidas foram obtidas na altura do peito $(1,3 \mathrm{~m})$.

Os dados de abertura do dossel tiveram que ser transformados para o inverso do seno da hipérbole para normalizarem (D'Agostino - Pearson, $g l=2, P=0,336$ ), por possuírem distribuição binomial negativa (Zar 1999).

Análise dos dados - Os dados de produção de serapilheira foram analisados por Anova de 2 fatores (tamanho de fragmento e posição no fragmento: borda $\mathrm{x}$ interior) com medidas repetidas, medidas dependentes do tempo (von Ende 1993). As análises foram conduzidas no programa Statistica versão 6.0 (Statsoft 2001).

Os dados de espessura da camada de serapilheira e de abertura do dossel foram analisados por Anova de três fatores (tamanho de fragmento, posição no fragmento: borda $\mathrm{x}$ interior e época coletada) e o teste usado a posteriori foi o de Tukey (Zar 1999), ambos conduzidos no programa Systat versão 8.0 (Systat 1998).

O teste de normalidade de D“Agostino-Pearson para $n$ $\geq 20$ para todos os dados analisados foi conduzido no programa Bioestat versão 2.0 (Ayres et al. 2000).

\section{Resultados}

Produção de serapilheira - Foram retirados das análises 12 dos 448 dados coletados, 11 dos quais eram "outliers" por serem constituídos, basicamente, por galhos muito grandes. $\mathrm{O}$ outro era constituído por um pouco mais da sua metade por folhas, provavelmente por estar muito próximo de uma árvore caducifólia ou de uma árvore que morreu durante o estudo. Seis das 12 vezes ocorreram na estação chuvosa (coleta 2 ), cinco na estação seca (coleta 4) e uma no final da estação chuvosa e início da estação seca (coleta 3). Ocorreram 8 vezes na borda e 4 no interior dos fragmentos e ocorreram de maneira igual no fragmento grande e nos pequenos.

A produção de serapilheira dependeu do tamanho dos fragmentos estudados $(F=3,27, g l=5, P=0,01$, tabela 1$)$, havendo uma tendência do maior fragmento apresentar maior produção (figura 2). De maneira geral, não foi encontrada diferença na produção entre borda e interior $(F=3,62, g l=1, P=0,06$, figura 2$)$, porém um fragmento pequeno (30 ha) apresentou maior produção 
na borda do que no interior $(F=3,59, g l=5, P=0,01)$. A mediana da produção de serapilheira anual no interior do fragmento grande foi de $8,90 \mathrm{t} \mathrm{ha}^{-1} \mathrm{ano}^{-1}$ (mínimo 3,93 e máximo $17,71 \mathrm{t} \mathrm{ha}^{-1}$ ano $^{-1}$ ) e na borda do mesmo fragmento foi de 8,76 $\mathrm{t} \mathrm{ha}^{-1}$ ano $^{-1}$ (mínimo 3,03 e máximo $16,50 \mathrm{t} \mathrm{ha}^{-1}$ ano $^{-1}$ ). Nos três fragmentos pequenos a mediana da produção de serapilheira anual no interior foi de 7,26 t ha ${ }^{-1}$ ano $^{-1}$ (mínimo 1,73 e máximo 13,11 t ha ${ }^{-1}$ ano $^{-1}$ ) e na borda foi de $7,27 \mathrm{t} \mathrm{ha}^{-1}$ ano $^{-1}$ (mínimo 4,43 e máximo $15,98 \mathrm{t} \mathrm{ha}^{-1} \mathrm{ano}^{-1}$ ). A produção de serapilheira também dependeu da estação estudada $(F$ $=9,59, g l=3, P<0,001)$. No final da estação seca e início da chuvosa (coleta 1 , mediana $=1,83 \mathrm{t} \mathrm{ha}^{-1}$ ) foi encontrada a menor produtividade de serapilheira. $\mathrm{Na}$ estação chuvosa (coleta 2 , mediana $=2,05 \mathrm{t} \mathrm{ha}^{-1}$ ) e no final da estação chuvosa e início da estação seca (coleta 3 , mediana $=2,03 \mathrm{t} \mathrm{ha}^{-1}$ ) foi encontrada uma produção intermediária e a maior queda de serapilheira ocorreu na estação seca (coleta 4 , mediana $\left.=2,33 \mathrm{t} \mathrm{ha}^{-1}\right)$ (figura 3). Esse padrão só foi significativo para um fragmento pequeno (18ha) $(F=2,31, g l=15, P<0,001)$, porém todos apresentaram a mesma tendência ao padrão encontrado nas diferenças entre as coletas. Não foram encontradas outras interações significativas dentre as testadas para a produção de serapilheira (tabela 1).

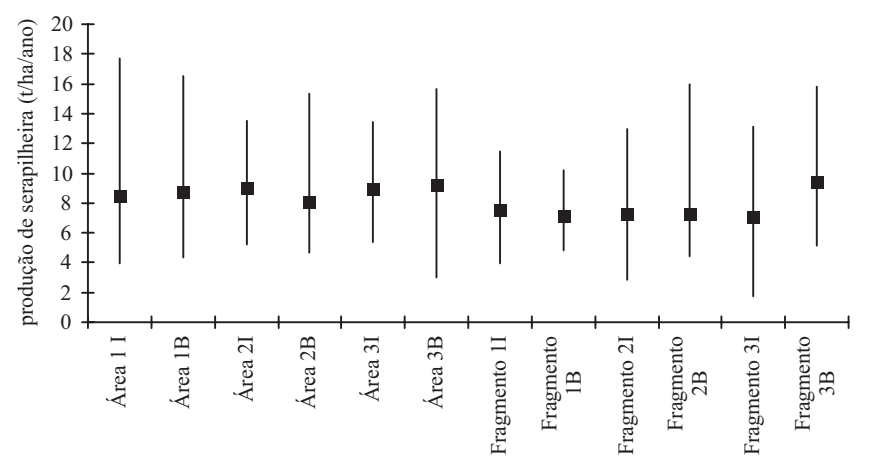

Figura 2. Mediana (ם), mínimo e máximo da produção de serapilheira anual ( $\left.\mathrm{t} \mathrm{ha-1} \mathrm{ano}^{-1}\right)$ nas seis áreas de estudo na região dos municípios de Cotia e Ibiúna, Estado de São Paulo, Brasil. Áreas 1, 2 e 3 referem-se às 3 réplicas da Reserva Estadual do Morro Grande; fragmento $1=14$ ha; fragmento $2=18$ ha; fragmento $3=30$ ha $(\mathrm{I}=$ interior, $\mathrm{B}=$ borda).

Figure 2. Median (ם), minimum and maximum annual litter production $\left(\mathrm{t} \mathrm{ha}^{-1}\right.$ year $\left.{ }^{-1}\right)$ in the six study areas in the region of the cities of Cotia and Ibiúna, State of São Paulo, Brazil. Areas 1, 2 and 3 are replications of the "Reserva Estadual do Morro Grande"; fragmento $1=14$ ha; fragmento $2=18$ ha; fragmento $3=30$ ha $(\mathrm{I}=$ interior, $\mathrm{B}=$ edge $)$.
Espessura da serapilheira - A espessura da camada de serapilheira dependeu do fragmento estudado $(F$ $=3,511, g l=5, P=0,004)$, mas não apresentou relação com o tamanho (figura 4). A diferença encontrada entre borda e interior quanto à espessura de serapilheira, dependeu do fragmento $(F=26,297, g l$ $=5, P<0,001)$, mas não apresentou relação com o tamanho do fragmento. O único fragmento que apresentou diferença entre a borda e interior, foi o de 30 ha, apresentando maior espessura da serapilheira na borda. A mediana da espessura de serapilheira no interior do fragmento grande foi de $3,5 \mathrm{~cm}$ (mínimo 0,50 e máximo $9,0 \mathrm{~cm}$ ), na borda do mesmo fragmento foi de $3,2 \mathrm{~cm}$ (mínimo 0,0 e máximo $8,0 \mathrm{~cm}$ ). Nos fragmentos pequenos a mediana da espessura de serapilheira no interior foi de $3,0 \mathrm{~cm}$ (mínimo 0,5 e máximo $30,0 \mathrm{~cm}$ ) e na borda foi de 4,1 cm (mínimo 0,5 e máximo 16,0 cm). A diferença encontrada entre as estações, também dependeu do fragmento $(F=8,170, g l=5, P<0,001)$, mas também não apresentou relação com o tamanho do fragmento. De modo geral, bordas e interiores apresentaram a mesma espessura de serapilheira (exceto para o fragmento de $30 \mathrm{ha}$ ), assim como as diferentes estações do ano (tabela 2).

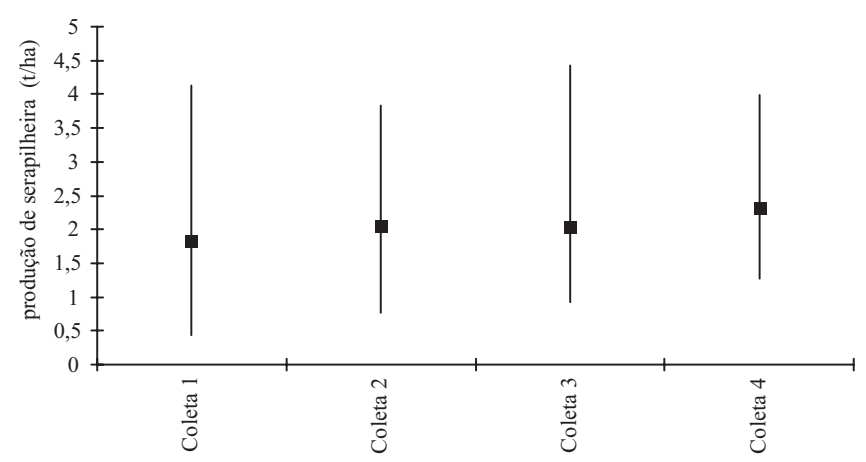

Figura 3. Mediana (ם), mínimo e máximo da produção trimestral de serapilheira (t.ha-1) com os dados das seis áreas de estudo (borda e interior) nas quatro coletas de dado na região dos Municípios de Cotia e Ibiúna, Estado de São Paulo, Brasil. Coleta 1 - dezembro de 2000, Coleta 2 - março de 2001, Coleta 3 - junho de 2001 e Coleta 4 - setembro de 2001.

Figure 3. Median ( $\square$ ), minimum and maximum quarterly litter production (t.ha ${ }^{-1}$ ) with the data of the six study areas (edge and interior) in the four collections in the region of the cities of Cotia and Ibiúna, State of São Paulo, Brazil. Collection 1 December of 2000, Collection 2 - March of 2001, Collection 3 - June of 2001 and Collection 4 - September of 2001. 
Abertura do dossel - Os fragmentos aqui estudados apresentaram diferenças quanto à abertura do dossel mas não houve relação com o tamanho $(F=4,845, g l=$ $5, P=0,001$, figura 5). Uma das áreas do fragmento grande (Área 1 - mediana 11,52\%) e um fragmento pequeno (18 ha -9,89\%) apresentaram abertura do dossel maior que as demais áreas. Em termos gerais, bordas apresentaram maior abertura do dossel do que interiores de fragmentos $(F=5,711, g l=1, P=0,019)$, porém essa diferença dependeu do fragmento estudado $(F=7,184, g l=5, P<0,001)$. As áreas 1 e 2 do fragmento grande apresentaram os interiores com menor abertura do dossel do que as respectivas bordas, ocorrendo o inverso na área 3. Já os fragmentos

Tabela 1. ANOVA de medidas repetidas da variável dependente: produção de serapilheira (PS). Fatores independentes para a ANOVA de medidas repetidas: $\mathrm{F}=$ fragmentos; $\mathrm{B}$ x I = borda $\mathrm{x}$ interior e $\mathrm{C}=$ coleta (tempo) como a medida repetida. As interações são representadas por *. Resultados significativos $(P<0,05)$ estão em negrito.

Table 1. Repeated measures ANOVA. Dependent variable: litter production (PS). Independent factors for the repeated measures ANOVA: $\mathrm{F}$ = fragments; $\mathrm{B}$ x I = edge $\mathrm{x}$ interior and $\mathrm{C}=$ collection (time) as repeated measure. The interactions are represented by *. Significant results $(P<0,05)$ are in boldface.

\begin{tabular}{|c|c|c|c|c|c|c|c|c|c|}
\hline & \multicolumn{3}{|c|}{$\bar{F}$} & \multicolumn{3}{|c|}{$\mathrm{B} \times \mathrm{I}$} & \multicolumn{3}{|c|}{$\mathrm{C}$} \\
\hline & $F$ & $g l$ & $P$ & $F$ & $g l$ & $P$ & $F$ & $g l$ & $P$ \\
\hline$P S$ & 3,27 & 5 & 0,01 & 3,62 & 1 & 0,06 & 9,59 & 3 & $<0,001$ \\
\hline
\end{tabular}

\begin{tabular}{|c|c|c|c|c|c|c|c|c|c|}
\hline & \multicolumn{3}{|c|}{$\mathrm{F}^{*} \mathrm{~B} \times \mathrm{I}$} & \multicolumn{3}{|c|}{$\mathrm{F}^{*} \mathrm{C}$} & \multicolumn{3}{|c|}{$\mathrm{B} \times \mathrm{I} * \mathrm{C}$} \\
\hline & $F$ & $g l$ & $P$ & $F$ & $g l$ & $P$ & $F$ & $g l$ & $P$ \\
\hline PS & 3,59 & 5 & $\mathbf{0 , 0 1}$ & 2,31 & 15 & $<0,001$ & 1,94 & 3 & 0,21 \\
\hline
\end{tabular}

\begin{tabular}{cccc}
\hline & \multicolumn{3}{c}{$\mathrm{F}^{*} \mathrm{~B} \times \mathrm{I} * \mathrm{C}$} \\
\cline { 2 - 4 } & $F$ & $g l$ & $P$ \\
\hline PS & 1,65 & 15 & 0,06 \\
\hline
\end{tabular}

Tabela 2. ANOVA de três fatores (AD e PrS). Variáveis dependentes: AD = abertura do dossel e PrS = espessura da serapilheira. Fatores independentes para a ANOVA de três fatores: $\mathrm{F}=$ fragmentos; $\mathrm{B}$ x I $=$ borda $\mathrm{x}$ interior e $\mathrm{C}=$ coleta (tempo). As interações são representadas por *. Resultados significativos $(P<0,05)$ estão em negrito.

Table 2. Three factors ANOVA (AD and PrS). Dependent variable: AD = canopy openness, PrS = thickness of the litter. Independent factors for three factors ANOVA: $\mathrm{F}=$ fragments; $\mathrm{B}$ x I $=$ edge $\mathrm{x}$ interior and $\mathrm{C}=$ collection (time). The interactions are represented by $*$. Significant results $(P<0,05)$ are in boldface.

\begin{tabular}{|c|c|c|c|c|c|c|c|c|c|}
\hline & \multicolumn{3}{|c|}{$\mathrm{F}$} & \multicolumn{3}{|c|}{$\mathrm{B} \times \mathrm{I}$} & \multicolumn{3}{|c|}{$\mathrm{C}$} \\
\hline & $F$ & $g l$ & $P$ & $F$ & $g l$ & $P$ & $F$ & $g l$ & $P$ \\
\hline $\mathrm{AD}$ & 4,845 & 5 & 0,001 & 5,711 & 1 & 0,019 & 39,112 & 1 & $<0,001$ \\
\hline $\operatorname{PrS}$ & 3,511 & 5 & 0,004 & 0,987 & 1 & 0,321 & 0,027 & 1 & 0,869 \\
\hline
\end{tabular}

\begin{tabular}{|c|c|c|c|c|c|c|c|c|c|}
\hline & \multicolumn{3}{|c|}{$\mathrm{F}^{*} \mathrm{~B} \times \mathrm{I}$} & \multicolumn{3}{|c|}{$\mathrm{F} * \mathrm{C}$} & \multicolumn{3}{|c|}{$\mathrm{B} \times \mathrm{I} * \mathrm{C}$} \\
\hline & $F$ & $g l$ & $P$ & $F$ & $g l$ & $P$ & $F$ & $g l$ & $P$ \\
\hline $\mathrm{AD}$ & 7,184 & 5 & $<0,001$ & 1,567 & 5 & 0,177 & 3,362 & 3 & 0,070 \\
\hline $\operatorname{PrS}$ & 26,297 & 5 & $<0,001$ & 8,170 & 5 & $<0,001$ & 0,285 & 1 & 0,594 \\
\hline
\end{tabular}

\begin{tabular}{cccc}
\hline & \multicolumn{3}{c}{$\mathrm{F}^{*} \mathrm{~B} \times \mathrm{I}^{*} \mathrm{C}$} \\
\cline { 2 - 4 } & $F$ & $g l$ & $P$ \\
\hline $\mathrm{AD}$ & 0,921 & 15 & 0,471 \\
$\mathrm{PrS}$ & 2,082 & 5 & 0,065 \\
\hline
\end{tabular}




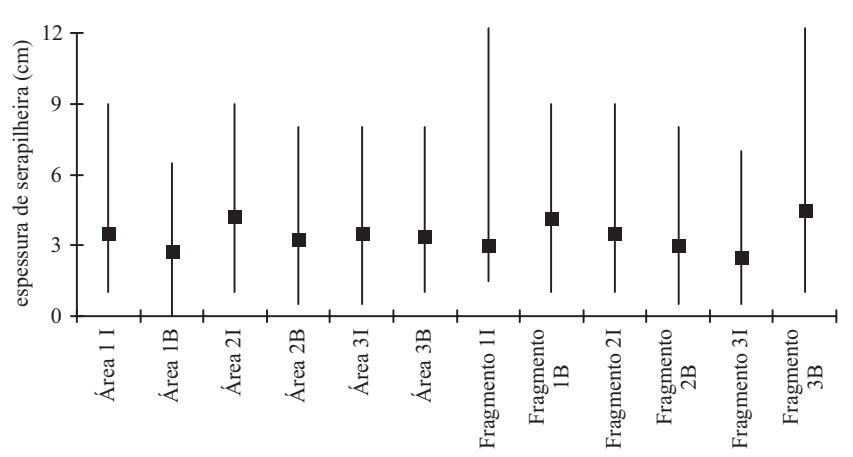

Figura 4. Mediana ( $\square$ ), mínimo e máximo da espessura de serapilheira $(\mathrm{cm})$ nas seis áreas de estudo na região dos municípios de Cotia e Ibiúna, Estado de São Paulo, Brasil. Áreas 1, 2 e 3 referem-se às 3 réplicas da Reserva Estadual do Morro Grande; fragmento $1=14$ ha; fragmento $2=18$ ha; fragmento 3 possui 30 ha $(\mathrm{I}=$ interior, $\mathrm{B}=$ borda). Valores máximos não mostrados no gráfico: fragmento $1 \mathrm{I}=30 \mathrm{~cm}$, fragmento $3 \mathrm{~B}=16 \mathrm{~cm}$.

Figure 4. Median (ם), minimum and maximum thickness of litter $(\mathrm{cm})$ in the six study areas in the region of the cities of Cotia and Ibiúna, State of São Paulo, Brazil. Areas 1, 2 and 3 are replications of the "Reserva Estadual do Morro Grande"; fragmento $1=14$ ha; fragmento $2=18$ ha; fragmento $3=30$ ha ( $\mathrm{I}=$ interior, $\mathrm{B}=$ edge). Maximum values not shown in the graph: Fragment $1 \mathrm{I}=30 \mathrm{~cm}$, Fragment $3 \mathrm{~B}=16 \mathrm{~cm}$.

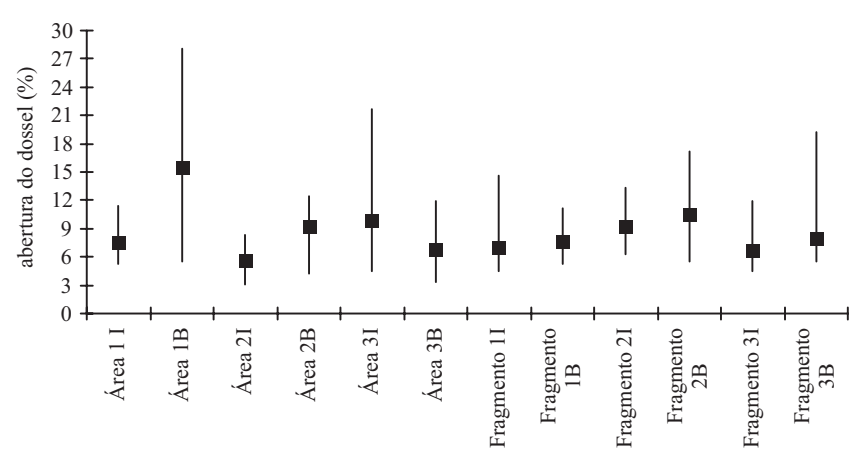

Figura 5. Mediana (ם), mínimo e máximo da abertura do dossel nas seis áreas de estudo na região dos municípios de Cotia e Ibiúna, Estado de São Paulo, Brasil. Áreas 1, 2 e 3 referem-se às 3 réplicas da Reserva Estadual do Morro Grande; fragmento $1=14$ ha; fragmento $2=18$ ha; fragmento $3=30$ ha $(\mathrm{I}=$ interior, $\mathrm{B}=$ borda)

Figure 5. Median ( $\boldsymbol{\square})$, minimum and maximum canopy openness in the six study areas in the region of the cities of Cotia and Ibiúna, State of São Paulo, Brazil. Areas 1, 2 and 3 are mentioned as reply of the "Reserva Estadual do Morro Grande"; fragmento $1=14 \mathrm{ha}$; fragmento $2=18 \mathrm{ha}$; fragmento $3=30$ ha $(\mathrm{I}=$ interior, $\mathrm{B}=$ edge $)$ pequenos não apresentaram diferenças de abertura do dossel entre a borda e o interior. A mediana da abertura do dossel no interior do fragmento grande foi de 7,55\% (mínimo 3,13\% e máximo 21,61\%) e na borda do mesmo fragmento foi de $9,25 \%$ (mínimo 3,39\% e máximo $28,13 \%)$. Nos fragmentos pequenos a mediana da abertura do dossel no interior foi de 7,03\% (mínimo $4,43 \%$ e máximo $14,58 \%$ ) e na borda foi de $7,94 \%$ (mínimo 5,21\% e máximo 19,27\%). A amostra de setembro de 2001 (mediana $=9,63 \%$ ) apresentou o dossel quase duas vezes mais aberto do que a amostra de dezembro de 2001 (mediana $=5,99 \%)(F=39,112$, $g l=1, P<0,001)$. Não foi encontrada significância nas outras interações testadas (tabela 2).

\section{Discussão}

No fragmento grande, a quantidade de serapilheira produzida foi maior que nos fragmentos pequenos. Essa diferença deve estar relacionada com a maior densidade de árvores (DAP $>5 \mathrm{~cm}$ ), maior área basal e maior porte das árvores no fragmento grande (Durigan et al. dados não publicados), podendo esse fato ser um indicativo do estado de regeneração mais avançado em que esta área se encontra. Em dois trabalhos realizados na Amazônia em fragmentos criados experimentalmente (Scariot 2000, Sizer et al. 2000) os fragmentos apresentaram queda de serapilheira semelhante àquela observada em áreas de floresta contínua. Certamente a diferença entre os resultados encontrados aqui e os da Amazônia se deve aos diferentes históricos das duas regiões, principalmente com relação à idade e ao tamanho das áreas.

Bordas antigas, aparentemente, têm sua influência negativa no interior do fragmento diminuída à medida em que a vegetação cresce (MacDougall \& Kellman 1992). Essa parece ser a razão dos resultados encontrados, pois os fragmentos estudados possuem borda há, pelo menos, 50 anos e, portanto, a borda já pode ter se recuperado. Outros estudos encontraram maior produção de serapilheira na borda do que no interior de fragmentos florestais, sendo tal diferença atribuída a diferenças na composição da vegetação e de microclima entre borda e interior (Williams-Linera 1990, Kapos et al. 1993, Matlack 1993, Kapos et al. 1997, Viana et al. 1997, Vasconcelos \& Luizão 2004). Esse fato também pode estar relacionado à maior mortalidade de árvores em bordas de fragmentos (Mesquita et al. 1999, Laurance et al. 2001). Outro estudo realizado na Mata Atlântica, em uma borda de 
aproximadamente 40 anos, encontrou resultado contrário, com maior produção no interior do fragmento (Nascimento 2005). Portanto, o efeito da borda na produção de serapilheira parece não seguir um padrão, podendo depender de inúmeros fatores tais como tipo de floresta, histórico da fragmentação, tamanho e grau de isolamento dos fragmentos e tipo de matriz circundante, entre outros.

A produção total de serapilheira por ano encontrada aqui está dentro dos valores encontrados para as florestas tropicais da América do Sul (Nascimento 2005). Segundo a revisão feita por Nascimento (2005), áreas da Mata Atlântica em estádio mais inicial de regeneração possuem menor produção de serapilheira do que aquelas em estádio mais avançado. Esse padrão parece ter se repetido neste estudo, onde o fragmento grande, em melhor estado de conservação, produziu mais serapilheira que os fragmentos pequenos.

A variação temporal na queda de serapilheira é correlacionada com a quantidade de chuvas, sendo relatada queda contínua, de intensidade variável, com um máximo de queda no final da estação seca, para as florestas tropicais sazonais do mundo. Aqui, o padrão encontrado foi de maior produção de serapilheira na estação seca, certamente infuenciado pela grande diminuição das chuvas no período seco. Já a queda de galhos (os "outliers") ocorreu com maior intensidade na época das chuvas, pois chuvas fortes podem provocar perturbação no interior da floresta, e na época da seca, em que a floresta está mais aberta pela queda de folhas e assim o vento penetra mais profundamente no interior da floresta.

Espessura da serapilheira - Não foi encontrada influência na espessura da serapilheira em função do tamanho do fragmento e nem da proximidade da borda. De modo geral, a espessura da camada de serapilheira de uma área está ligada diretamente à produção de serapilheira e à velocidade de decomposição, que influencia o acúmulo desta sobre o solo da floresta. Entretanto, como foi encontrada maior produção de serapilheira no maior fragmento e a espessura de serapilheira não variou com o tamanho do fragmento, certamente esse fato se deve a uma maior taxa de decomposição no maior fragmento, fato já relatado na literatura para fragmentos na Amazônia e explicado pela maior umidade encontrada em fragmentos mais conservados (Didham 1998).

Abertura do dossel - Alguns estudos observaram que o grau de abertura do dossel de uma floresta pode ser um indicativo de perturbação da área (Kapos 1989, WilliamsLinera 1990, MacDougall \& Kellman 1992, Nicotra et al. 1999). Os fragmentos aqui estudados não apresentaram um padrão de abertura do dossel ligado ao tamanho e à proximidade da borda. Em um estudo realizado em fragmentos de 1 e 100 ha na Amazônia, essa relação foi observada, com o menor fragmento apresentando uma maior intensidade luminosa em seu interior do que o maior (Kapos 1989). A falta de relação entre o tamanho de fragmento e a proximidade da borda com abertura do dossel encontrada no presente trabalho, parece indicar uma grande heterogeneidade espacial na entrada de luz na floresta, que pode estar relacionada com a freqüência de clareiras e com a complexidade estrutural e deciduidade da floresta (Bianchini et al. 2001). Ou seja, a heterogeneidade pode estar influenciando mais na entrada de luz na floresta do que o tamanho do fragmento e a influência da borda. Esse resultado pode, também, estar relacionado com a idade dos fragmentos aqui estudados e com a diferença de tamanho dos fragmentos estudados na Amazônia e os daqui.

De acordo com os resultados encontrados, a produção de serapilheira foi maior no maior fragmento estudado, entretanto as bordas dos fragmentos não influenciaram na produção de serapilheira. A estação seca teve influência direta no aumento da produção de serapilheira. Já a espessura da camada de serapilheira depositada sobre o solo não foi influenciada pelo tamanho do fragmento e não dependeu da proximidade da borda. A abertura do dossel da floresta não apresentou relação com o tamanho do fragmento e não foi encontrado um padrão com relação à influência da borda. Com os resultados relacionados à área do fragmento, pode-se inferir que a diferença entre queda de serapilheira e acúmulo no solo deve estar relacionada com diferenças de decomposição nesses fragmentos, sendo maior no fragmento grande devido à maior umidade. Já uma relação entre produção de serapilheira e abertura do dossel não foi encontrada, provavelmente devido à grande heterogeneidade espacial causada pela freqüência de clareiras e complexidade estrutural e deciduidade da floresta.

Este estudo permitiu observar que existem algumas diferenças entre fragmentos e com relação à proximidade da borda, em todas as variáveis aqui estudadas, porém, muitas vezes, essas diferenças não apresentaram relação direta com o tamanho do fragmento e parecem ser específicas para cada área. Como florestas tropicais são mosaicos dinâmicos, talvez não exista um padrão quanto aos efeitos da fragmentação, mas a produção e acúmulo de serapilheira dependem das características e da dinâmica de cada floresta. 
Agradecimentos - À Sabesp por permitir o trabalho na Reserva Estadual do Morro Grande, aos donos dos fragmentos por permitir o trabalho, ao R. Belinello, L. F. Alves, B. Canela e D. Bertani pela ajuda no campo, ao L. F. Moraes pelos comentários a versão preliminar do manuscrito e ao $\mathrm{P}$. Cavalin pela ajuda com a estatística. À Fapesp (Proc. $n^{\text {os }} .99 /$ 05123-4 e 00/00596-0) pelo financiamento. F.A.M. Santos agradece ao Conselho Nacional de Desenvolvimento Científico e Tecnológico $(\mathrm{CNPq})$ pela bolsa de produtividade em pesquisa (307132/2004-8). Este estudo é parte do projeto "Conservação da Biodiversidade em uma paisagem fragmentada no Planalto Atlântico de São Paulo - Biota/ Projeto Caucaia".

\section{Referências bibliográficas}

ALVAREZ-SÁNCHEZ, J. \& GUEVARA, S. 1999. Litter interception on Astrocaryum mexicanum Liebm. (Palmae) in a Tropical Rain Forest. Biotropica 31:89-92.

AYRES, M., AYRES JUNIOR., M., AYRES, D.L. \& SANTOS, A.S.S. 2000. BioEstat versão 2. Sociedade Civil Mamirauá, Belém.

BENÍTEZ-MALVIDO, J. \& MARTINEZ-RAMOS, M. 2003. Impact of forest fragmentation on understory plant species richness in Amazonia. Conservation Biology 17:389-400.

BERNACCI, L.C., DURIGAN, G., CORREIA, G., ARBOCZ, G., CATHARINO, E. \& METZGER, J.P.M. 2004. Composição florística e estrutura da vegetação em fragmentos florestais do Planalto de Ibiúna. Anexo 7. In Conservação da biodiversidade em paisagens fragmentadas no Planalto Atlântico de São Paulo (J.P. Metzger, ed.). Fapesp 99/ 05123-4 technical report n.4, Fapesp, São Paulo.

BIANCHINI, E., PIMENTA, J.A. \& SANTOS, F.A.M. 2001. Spatial and temporal variation in the canopy cover in a tropical semi-deciduous forest. Brazilian Archives of Biology and Technology 44:269-276.

BRUNA, E.M. 2002. Effects of forest fragmentation on Heliconia acuminata seedling recruitment in central Amazonia. Oecologia 132:235-243.

DIDHAM, R.K. \& LAWTON, J.H. 1999. Edge structure determines the magnitude of changes in microclimate and vegetation structure in Tropical Forest fragments. Biotropica 31:17-30.

DIDHAM, R.K. 1998. Altered leaf-litter decomposition rates in tropical forest fragments. Oecologia 116:397-406.

DRAKE, D.R. \& PRATT, L.W. 2001. Seedling mortality in Hawaiian rain forest: the role of small-scale physical disturbance. Biotropica 33:319-323.

EDWARDS, P.J. 1977. Studies of mineral cycling in a montane rain forest in New Guinea. Journal of Ecology 65:971-992.

FACCELLI, J.M. \& FACCELLI, E. 1993. Interactions after death: plant litter controls priority affects in a successional plant community. Oecologia 95:277-282.
FACCELLI, J.M. \& PICKETT, S.T.A. 1991. Plant litter: its dynamics and effects on plant community structure. The Botanical Review 57:1-32.

FONTOURA, S.B., GANADE, G. \& LAROCCA, J. 2006. Changes in plant community diversity and composition across an edge between Araucaria forest and pasture in South Brazil. Revista Brasileira de Botânica 29:79-91.

FOX, B.J., TAYLOR, J.E., FOX, M.D. \& WILLIAMS, C. 1997. Vegetation changes across edges of rainforest remnants. Biological Conservation 82:1-13.

GARCÍA-GUZMÁN, G. \& BENÍTEZ-MALVIDO, B. 2003. Effect of litter on the incidence of leaf-fungal pathogens and herbivory in seedling of the tropical tree Nectandra ambigens. Journal of Tropical Ecology 19:171-177.

GEHLHAUSEN, S.M., SCHWARTZ, M.W. \&AUGSPURGER, C.K. 2000. Vegetation and microclimatic edge effects in two mixed-mesophytic forest fragments. Plant Ecology 147:21-35.

HAINES, B. \& FOSTER, R.B. 1997. Energy flow through litter in a Panamanian forest. Journal of Ecology 65:147-155.

KAPOS, V. 1989. Efffects of isolation on the water status of forest patches in the Brazilian Amazon. Journal of Tropical Forest 5:173-185.

KAPOS, V., GANADE, G., MATSUI, E. \& VICTORIA, R.L. 1993. $\mathrm{d}^{13} \mathrm{C}$ as an indicator of edge effects in tropical rainforest reserves. Journal of Ecology 81:425-432.

KAPOS, V., WANDELLI, E., CAMARGO, J.L. \& GANADE, G. 1997. Edge- related changes in environment and plant responses due to forest fragmentation in Central Amazonia. In Tropical forest remnants: Ecology, management, and conservation of fragmented communities (W. F. Laurance \& R.O. Bierregaard, eds.). Chicago University Press, New York, p.33-44.

KÖPPEN, W. 1948. Climatologia. Fondo de Cultura Económica, México.

KUNKEL-WESTPHAL, I. \& KUNKEL, P. 1979. Litter fall in a Guatemalan primary forest, with details of leaf-shedding by some common tree species. Journal of Ecology 67:665-686.

LAURANCE, W.F., SALICRUP, D.P., DELAMÔNICA, P., FEARNSIDE, P.M., D’ANGELO, S., JEROZOLINSKI, A., POHL, L. \& LOVEJOY, T.E. 2001. Rain Forest fragmentation and structure on Amazonian liana communities. Ecology 82:105-116.

LAURANCE, W.F. \& YENSEN, E. 1991. Predicting the impacts of edge effects in fragmented habitats. Biological Conservation 55:77-92.

MACDOUGALL, A. \& KELLMAN, M. 1992. The understorey light regime and patterrns of tree seedlings in tropical riparian forest patches. Journal of Biogeography 19:667675.

MARTÍNEZ-RAMOS, M., ALVAREZ-BUYLLA, E., SARUKHÁN, J. \& PIÑERO, D. 1988. Treefall age determination and gap dynamics in a tropical forest. Journal of Ecology 76:700-716. 
MATLACK, G.R. 1993. Microenvironment variation within and among forest edge sites in the Eastern United States. Biological Conservation 66:185-194.

MESQUITA, R.C.G., DELAMÔNICA, P. \& LAURENCE, W.F. 1999. Effect of surrounding vegetation on edge-related tree mortality in Amazonian forest fragments. Biological Conservation 91:129-134.

METZGER, J.P., SIMONETTI, C., UEZU,A., DIEDERICHSEN, A. \& GOULART, W. 2002. Estrutura da paisagem em Caucaia do Alto (Planalto de Ibiúna, SP). Sub-projeto 1. Composição florística e estrutura da vegetação. Relatório Fapesp de acompanhamento anual do programa Biota/Fapesp: Biodiversity conservation in fragmented landscape at the Atlantic Plateau of São Paulo (Brazil). Processo No 99/05123-4.

MORAES, R.M., DELITTI, W.B.C. \& DE VUONO, Y.S. 1999. Litterfall and litter nutrient content in two Brazilian tropical forests. Revista Brasileira de Botânica 22:9-16.

MORELLATO, L.P.C. 1992. Nutrient cycling in two southeast Brazilian forests: litterfall and litter standing crop. Journal of Tropical Ecology 8:205-215.

NASCIMENTO, A.C.P. 2005. Produção e aporte de nutrientes da serrapilheira em um fragmento de mata atlântica na Rebio União, RJ: efeito de borda. Dissertação de mestrado, Universidade Estadual do Norte Fluminense Darcy Ribeiro, Campos dos Goytacazes.

NICOTRA, A.B., CHAZDON, R.L. \& IRIARTE, S.V.B. 1999. Spatial heterogeneity of light and wood seedling regeneration in tropical wet forests. Ecology 80:19081926.

OLIVEIRAFILHO, A.T. \& FONTES, M.A.L. 2000. Patterns of floristic differentiation among Atlantic Forests in Southeastern Brazil and the influence of climate. Biotropica 32:793-810.

PARDINI, P., SOUZA, S.M., BRAGANETO, R. \& METZGER, J.P. 2005. The role of forest structure, fragment size and corridors in maintaining small mammal abundance and diversity in an Atlantic forest landscape. Biological Conservation 124:253-266.

READER, R.J. 1993. Control on seedling emergence by ground cover and seed predation in relation to seed size for some old-field species. Journal of Ecology 81:169-175.

ROGERS, H.M. 2002. Litterfall, decomposition and nutrient release in a lowland tropical rain forest, Morobe Province, Papua New Guinea. Journal of Tropical Ecology 18:449-456.

ROSS, J.L.S. \& MOROZ, I.C. 1997. Mapa geomorfológico do Estado de São Paulo, escala 1:500.000. GeografiaFFLCH-USP, IPT/ Fapesp, São Paulo, v. 1.
SABESP. 1997. Programa de Conservação do Sistema Cotia. Relatório Conclusivo (tomo 3): Avaliação Ambiental. Sabesp/Fundação Brasileira para o Desenvolvimento Sustentável, São Paulo.

SANTOS, S. L. \& VÁLIO, I. F. M. 2002. Litter accumulation and its effect on seedling recruitment in a Southeast Brazilian Tropical Forest. Revista Brasileira de Botânica 25:89-92.

SCARIOT, A. 2000. Seedling mortality by litterfall in Amazonian forest fragments. Biotropica 32:662-669.

SIQUEIRA, L.P., MATOS, D.M.S., PORTELA, R.C.Q., BRAZ, M.I.G., SILVA-LIMA, L. \& MATOS, M. B. 2004. Using the variances of microclimate variables to determine edge effects in small forest Atlantic Rain Forest fragments, South-Eastern Brazil. Ecotropica 10:59-64.

SIZER, N., TANNER, E.V.J. \& FERRAZ, I.D.K. 2000. Edge effects on litterfall mass and nutrient concentrations in forest fragments in central Amazonia. Journal of Tropical Ecology 16:853-863.

STATSOFT. 2001. STATISTICA (data analysis software system), version 6. www.statsoft.com.

SYSTAT. 1998. SYSTAT 8.0 Statistics. SPSS, Sao Jose, CA.

UEZU,A., METZGER, J.P. \& VIELLIARD, J.M.E. 2005. Effects of structural and functional connectivity and patch size on the abundance of seven Atlantic Forest bird species. Biological Conservation 123:507-519.

VASCONCELOS, H.L. \& LUIZÃO, F.J. 2004. Litter production and litter nutrient concentrations in a fragmented Amazonian landscape. Ecological Application 14:884892.

VIANA, V.M., TABANEZ, A.A.J. \& BATISTA, J.L. 1997. Dynamics and restoration of forest fragments in the Brazilian Atlantic Moist Forest. In Tropical forest remnants: Ecology, management, and conservation of fragmented communities (W.F. Laurance \& R.O. Bierregaard, eds.). Chicago University Press, New York, p.351-365.

VITOUSEK, P.M. 1984. Litterfall, nutrient cycling and nutrient limitation in tropical forest. Ecology 65:285-298.

VON ENDE, C.N. 1993. Repeated-measures analysis: growth and other time-dependent measures. In Design and analysis of ecological experiments (S. M. Scheiner \& J. Gurevitch, eds.). International Thomson Publishing, New York, p.113-137.

WILLIAMS-LINERA, G. 1990 Vegetation structure and environmental conditions of forest edges in Panama. Journal of Ecology 78:356-373.

ZAR, J. H. 1999. Biostatistical Analysis. $4^{\text {th }}$. Prentice Hall, New Jersey. 